\title{
Evaluation of variable numbers of tandem repeat as molecular epidemiological markers of Mycobacterium tuberculosis in Japan
}

Correspondence

Takayuki Wada

taka-wada@city.osaka.lg.jp

Received 6 October 2006

Accepted 16 March 2007

\section{Takayuki Wada, ${ }^{1}$ Shinji Maeda, ${ }^{2}$ Atsushi Hase ${ }^{1}$ and Kazuo Kobayashi ${ }^{3}$}

${ }^{1}$ Department of Microbiology, Osaka City Institute of Public Health and Environmental Sciences, 8-34 Tojo-cho, Tennoji-ku, Osaka 543-0026, Japan

${ }^{2}$ Molecular Epidemiology and Genetic Identification, Mycobacterium Reference Center, Research Institute of Tuberculosis, 3-1-24 Matsuyama, Kiyose, Tokyo 204-8533, Japan

${ }^{3}$ Department of Immunology, National Institute of Infectious Diseases, 1-23-1 Toyama, Shinjuku-ku, Tokyo 162-8640, Japan

\begin{abstract}
Using 243 Mycobacterium tuberculosis isolates obtained in 2001 in Osaka City, Japan, the discriminatory power of variable numbers of tandem repeats (VNTRs) of 12 standard mycobacterial interspersed repetitive units (MIRUs) was assessed. The biggest cluster defined by MIRU-VNTRs consisted of 57 (23.5\%) isolates and they belonged to the Beijing family based on spoligotyping. When additional VNTR loci were included in the MIRU-VNTR analysis, the 57 originally clustered strains were further differentiated by the addition of Queen's University Belfast (QUB)-VNTRs, but not exact tandem repeat-VNTR. The allelic diversity of additional VNTR loci such as VNTR 3232 (QUB-3232), VNTR 2163a (QUB-11a), VNTR 2163b (QUB-11b) and VNTR 1982 (QUB-18) was high in the 57 strains. When the 243 M. tuberculosis isolates were analysed using 16-locus VNTR (the 12 standard MIRUs and the 4 QUB loci) and IS6110 RFLP, the respective Hunter-Gaston discriminatory indexes were 0.9966 and 0.9971 . The discrimination power of 16-locus VNTR was equal to that of IS6110 RFLP analysis. If appropriate loci are added to the standard MIRU analysis, VNTR genotyping could be a valuable tool for strain typing and epidemiological research of $M$. tuberculosis in Japan.
\end{abstract}

\section{INTRODUCTION}

Tuberculosis $(\mathrm{TB})$ remains a major public health threat to the world population. The gold standard method for molecular epidemiological analyses for TB is IS6110 RFLP Southern blotting (Cave et al., 1991; Kremer et al., 1999; van Embden et al., 1993). However, it has some limitations, such as the long time for culturing TB bacilli, technical difficulties and the troublesome comparison of data obtained at different laboratories. Therefore, some alternative strategies and methods for epidemiological analysis of TB have been evaluated and reported recently.

Spoligotyping is the most popular PCR-based molecular analytical method in many countries (Kamerbeek et al., 1997). Nevertheless, this method is not informative for Mycobacterium tuberculosis Beijing family strains because

Abbreviations: ETR, exact tandem repeat; HGDI, Hunter-Gaston discriminatory index; MIRU, mycobacterial interspersed repetitive unit; QUB, Queen's University Belfast; TB, tuberculosis; VNTR, variable numbers of tandem repeat.

A table of VNTR profiles for the strains is available as supplementary material with the online version of this paper. almost all strains in this family share an identical spoligotyping pattern (Glynn et al., 2002; Kremer et al., 2004). Variable numbers of tandem repeat (VNTR) typing is also PCR-based. It amplifies various loci of minisatellites on a genome and enumerates their units as a molecular marker (Supply et al., 2000). In some reports, the discriminatory capacity of VNTR typing with 12 mycobacterial interspersed repetitive units (MIRUs) is comparable to that of IS6110 RFLP in North America and Europe (Blackwood et al., 2004; Mazars et al., 2001). The Centers for Disease Control and Prevention (CDC) in the USA have adopted it as a standard typing method (Cowan et al., 2005; CDC, 2004).

Although convenient genotyping of M. tuberculosis isolates has been achieved, the 12-locus MIRU-VNTR has yielded insufficient discrimination for Beijing strains. Therefore, analyses of various additional VNTR loci for such isolates have been undertaken (Kam et al., 2006; Kremer et al., 2005b; Nikolayevskyy et al., 2006; Surikova et al., 2005). Recently, novel standards, 15- or 24-loci MIRU-VNTR methods, were proposed based on analyses of strains from cosmopolitan origins (Supply et al., 2006). 
Beijing/W family has been reported to be predominantly spread throughout eastern Asia (Chan et al., 2001; van Soolingen et al., 1995). These strains are also present across former Soviet Union countries (Nikolayevskyy et al., 2006) and other areas, like the USA (Bifani et al., 2002). For application of the VNTR method to epidemiological analysis of M. tuberculosis in Japan, we performed IS6110 RFLP typing, spoligotyping and 12-locus standard or some additional locus VNTR analyses. These data might be useful to select appropriate VNTR loci for the genotyping of M. tuberculosis in Japan.

\section{METHODS}

Bacterial strains and DNA isolation. A total of $243 \mathrm{M}$. tuberculosis strains were collected at the Department of Microbiology, Osaka City Institute of Public Health and Environmental Sciences, from cultureconfirmed pulmonary TB patients who were reported to the public health centre of Osaka, Osaka City, Japan, between January 1 and December 31 2001. All the available isolates collected in the period were used for this study. They were obtained mainly from three hospitals and two medical centres in Osaka. The mean incidence of TB in Japan is 27.9 per 100000 population, but the incidence is much higher in Osaka City (82.6 per 100000 population). Mycobacterial genomic DNA was prepared from bacteria grown on Ogawa medium using a combination of phenol/chloroform extraction and mechanical disruption. The DNA concentration was estimated using UV absorbance at $260 \mathrm{~nm}$; then it was adjusted to $20 \mu \mathrm{g} \mathrm{ml}^{-1}$.

Molecular typing methods. IS6110 RFLP typing was performed according to a standardized protocol (van Embden et al., 1993). The band patterns were analysed using dedicated software (Molecular Analyst; Bio-Rad Laboratories). The spoligotyping method (Kamerbeek et al., 1997) was also performed according to a standard protocol (Molhuizen et al., 1998), except for the use of positively charged nylon membranes and DIG-labelled probes (Roche Diagnostic Systems) in place of biotin-labelled probes.

For this study, VNTR typing was performed with Ex Taq and GC PCR buffer I (TaKaRa Holdings). The sequences of the primers for amplification of 12 loci of MIRUs (MIRU-02, 04, 10, 16, 20, 23, 24, 26, 27, 31, 39 and 40), 4 loci of exact tandem repeats (ETRs) (ETR-A, $\mathrm{B}, \mathrm{C}$ and $\mathrm{F}$ ) and 8 loci of Queen's University Belfast (QUBs)-VNTR (QUB-11a, 11b, 15, 18, 23, 26, 1895, 3232 and 3336) were selected according to descriptions in other studies (Frothingham \& MeekerO'Connell, 1998; Kremer et al., 2005b; Skuce et al., 2002; Supply et al., 2001). The PCR mixture was prepared in a $20 \mu \mathrm{l}$ volume with $1 \times \mathrm{GC}$ PCR buffer I, $0.005 \mathrm{U}$ Ex Taq, $200 \mu \mathrm{M}$ each of the four dNTPs, $0.4 \mu \mathrm{M}$ each primer set and $10 \mathrm{ng}$ template DNA. The PCR conditions were as follows: initial denaturation at $95{ }^{\circ} \mathrm{C}$ for $10 \mathrm{~min}$, and then 35 cycles of $95{ }^{\circ} \mathrm{C}$ for $1 \mathrm{~min}, 60{ }^{\circ} \mathrm{C}$ for $1 \mathrm{~min}$ and $72{ }^{\circ} \mathrm{C}$ for $1 \mathrm{~min}$.
VNTR analysis of amplified DNA fragments. The sizes of amplified DNA fragments were determined using capillary array electrophoresis analysis equipment (i-chip SV1210; Hitachi Electronics Engineering). Using this equipment, sizes of DNA fragments were calculated digitally in six minutes. Their respective copy numbers were calculated from their size and assigned according to the number of repeats for each locus, and in agreement to published allelic tables (Supply et al., 2001) or as described elsewhere (Frothingham \& Meeker-O'Connell, 1998; Kremer et al., 2005b; Skuce et al., 2002; Supply et al., 2001). The accuracy of calculation was determined through comparison with a DNA ladder, as a fragmentsize standard, and confirmed through analysis of H37Rv.

Allelic diversity and discrimination. The allelic diversity $(h)$ at each VNTR locus was calculated using the equation $h=1-\Sigma x_{i}^{2}$, where $x_{i}$ is the frequency of the $i$ th allele at the locus, as used in previous works (Kremer et al., 2005b; Sun et al., 2004). The Hunter-Gaston discriminatory index (HGDI) was calculated in accordance with a method, explained in another paper, to evaluate the combination of some VNTR loci (Hunter \& Gaston, 1988).

\section{RESULTS}

\section{Genotyping analyses of total isolates}

All the 243 isolates were analysed using spoligotyping, MIRU-VNTR and IS6110 RFLP analyses. The numbers of clusters observed when performing IS6110 RFLP and 12loci MIRU-VNTR were roughly equal (27 in RFLP and 28 in VNTR) (Table 1). However, the respective percentages of clustering isolates by RFLP and VNTR analyses were 30.9 and $70.8 \%$. The biggest cluster sizes were $7(2.8 \%)$ for RFLP and $57(23.5 \%)$ for 12-locus MIRU-VNTR. The respective HGDI values of MIRU-VNTR and IS6110 RFLP were 0.928 and 0.997 . The discriminatory power of spoligotyping was lower (0.585), in the population, because it had no discrimination on 154 (63.4\% of strains) Beijing genotype isolates that shared an identical spoligotyping pattern. Moreover, 39 isolates were identified as Beijinglike strains based on the definition in a spoligotyping pattern proposed previously (Kremer et al., 2004; Supplementary Table S1 available with the online journal).

\section{Analyses of $\mathbf{5 7}$ MIRU-identical isolates}

Analyses by spoligotyping and MIRU-VNTR showed that the 57 isolates in the biggest MIRU-VNTR defined cluster belonged to the Beijing family (Supplementary Table S1 available with the online journal) and possessed identical

Table 1. Comparison of the discriminatory power of three molecular typing methods in 243 M. tuberculosis strains from Osaka City

\begin{tabular}{|lcccccc|}
\hline Typing method & $\begin{array}{c}\text { Total no. of type } \\
\text { patterns }\end{array}$ & $\begin{array}{c}\text { No. of unique } \\
\text { types }\end{array}$ & $\begin{array}{c}\text { No. of } \\
\text { clusters }\end{array}$ & $\begin{array}{c}\text { No. of clustered } \\
\text { isolates (\%) }\end{array}$ & $\begin{array}{c}\text { Maximum no. of isolates } \\
\text { in a cluster }\end{array}$ & HGDI* $^{*}$ \\
\hline IS6110 RFLP & 195 & 168 & 27 & $75(30.9)$ & 7 & 0.997 \\
Spoligotyping & 38 & 25 & 13 & $218(89.7)$ & 154 & 0.585 \\
12-loci MIRU-VNTR & 99 & 71 & 28 & $172(70.8)$ & 57 & 0.928 \\
\hline
\end{tabular}

${ }^{\star}$ HGDI was calculated as described by Hunter \& Gaston (1988). 
Table 2. The allelic profiles of each additional VNTR locus in $57 \mathrm{MIRU}$-identical strains

\begin{tabular}{|c|c|c|c|c|c|c|c|c|c|c|c|c|c|c|c|c|c|c|}
\hline VNTR locus & $\begin{array}{c}\text { No } \\
\text { amplification }\end{array}$ & 1 & 2 & 3 & 4 & 5 & 6 & 7 & 8 & 9 & 10 & 11 & 12 & 13 & 14 & 15 & 16 and over $\dagger$ & $h$ index \\
\hline VNTR 2165 (ETR A) & & 1 & 3 & 1 & 52 & & & & & & & & & & & & & 0.164 \\
\hline VNTR 2461 (ETR B) & & 2 & 51 & 3 & & & 1 & & & & & & & & & & & 0.132 \\
\hline VNTR 577 (ETR C) & & & & & 57 & & & & & & & & & & & & & 0.000 \\
\hline VNTR 2163b (QUB-11b) & & & 4 & 5 & 3 & 9 & 13 & 17 & 5 & 1 & & & & & & & & 0.811 \\
\hline VNTR 1982 (QUB-18) & & & & & & & & 6 & 19 & 1 & 28 & 3 & & & & & & 0.633 \\
\hline VNTR 1612 (QUB-23) & & & & & & 1 & 55 & 1 & & & & & & & & & & 0.068 \\
\hline VNTR 4052 (QUB-26) & & & & 2 & & & 2 & 3 & 41 & & 8 & 1 & & & & & & 0.457 \\
\hline VNTR 1895 (QUB-1895) & & & 5 & & 52 & & & & & & & & & & & & & 0.160 \\
\hline VNTR 3232 (QUB-3232) & & & & & & & 2 & & & & 2 & 1 & 9 & 6 & 14 & 8 & 15 & 0.851 \\
\hline
\end{tabular}

${ }^{\star} h$ index represents the allelic diversity of each locus, calculated as described in Methods.

$\dagger$ The number of strains that had more than 16 copies in the locus are represented all together in this column.

\$The deletion of $3^{\prime}$-terminal $24 \mathrm{bp}$ of a unit, which is present in nearly all isolates, was absent from this isolate.

MIRU genotypes (223325173533, sequentially in MIRU locus number). M. tuberculosis strains with this specific MIRU genotype accounted for $29.5 \%$ (57/193) of the Beijing and Beijing-like strains in Osaka. Using these MIRU-identical isolates, we analysed the discriminatory abilities of other VNTR loci. From the $h$ index, high allelic diversity among the 57 strains was observed at VNTR 3232 (QUB-3232), VNTR 2163a (QUB-11a), VNTR 2163b (QUB-11b) and VNTR 1982 (QUB-18) (Table 2). On the other hand, the four ETR loci that were the standard loci of additional VNTR analyses were insufficient to discriminate these strains and offered a low allelic diversity in this cluster (showing an $h$ index of 0 for ETR C and 0.306 for ETR F). Using our PCR conditions, the locus VNTR 3155 (QUB-15) could not be amplified (data not shown).

\section{Assessment of the combination of additional VNTR loci}

To confirm the combination of four QUB loci (VNTR 3232, VNTR 2163a, VNTR 2163b and VNTR 1982) as an additional VNTR loci set, their genotypes were compared with IS6110 RFLP analysis in 57 MIRU-identical strains. Among 11 clusters defined by the 4 QUB loci, 7 clusters were completely consistent with clusters identified using IS6110 RFLP analysis (data not shown). Isolates in each of the remaining four clusters showed nearly identical RFLP patterns (Fig. 1). A one band difference was observed in three clusters (clusters $\mathrm{A}, \mathrm{B}$ and $\mathrm{C}$ ) and the isolates in the remaining cluster $\mathrm{D}$ showed a two band difference. The discriminatory power of these four additional loci was

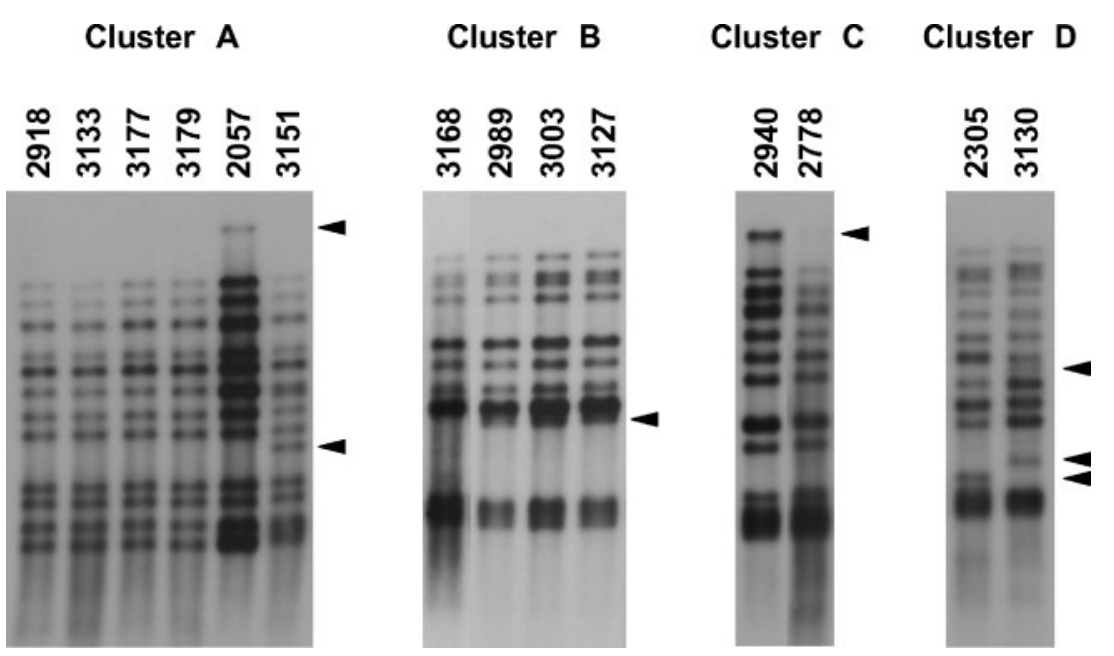

Fig. 1. Differences of IS6110 RFLP in isolates sharing the same QUB(4)-VNTR genotype. IS6110 RFLP genotypes were compared for four clusters (A, B, C and D) that represented the same QUB(4)-VNTR types but different IS6110 RFLP types in 57 MIRU-identical strains. Arrowheads indicate the differences in band pattern(s) of respective clusters. 
Table 3. The allelic profiles of four candidates for additional VNTR loci in 243 M. tuberculosis strains from Osaka City

\begin{tabular}{|c|c|c|c|c|c|c|c|c|c|c|c|c|c|c|c|c|c|c|}
\hline VNTR locus & $\begin{array}{c}\text { No } \\
\text { amplification }\end{array}$ & 1 & 2 & 3 & 4 & 5 & 6 & 7 & 8 & 9 & 10 & 11 & 12 & 13 & 14 & 15 & 16 and over $\dagger$ & $h$ index ${ }^{\star}$ \\
\hline VNTR 2163a (QUB-11a) & 13 & & 24 & & 9 & 22 & 3 & 22 & 102 & 35 & 1 & & & & & & 12 & 0.772 \\
\hline VNTR 2163b (QUB-11b) & 7 & 2 & 27 & 21 & 25 & 26 & 56 & 62 & 9 & 5 & & 2 & & & & & 1 & 0.837 \\
\hline VNTR 1982 (QUB-18) & & 2 & 8 & 9 & 2 & 23 & 5 & 16 & 51 & 16 & 104 & 7 & & & & & & 0.751 \\
\hline
\end{tabular}

${ }^{\star} h$ index represents the allelic diversity of each locus, calculated as described in Methods.

$\uparrow$ The number of strains that had more than 16 copies in the locus are represented all together in this column.

confirmed by analysing the remaining strains from the collection (186 isolates). The diversity of 4 loci, which offered high allelic diversity in 57 MIRU-identical strains, was also high in the 243 total strains (Table 3). We performed 16-loci VNTR [MIRU(12)+QUB(4)] analyses for the remaining $186 \mathrm{~TB}$ isolates to assess the efficiency of the addition of 4 loci to MIRU-VNTR. The VNTR profiles of all 243 isolates are listed in Supplementary Table S1 available with the online journal. The respective percentages of clustering isolates by RFLP and VNTR analyses were 30.9 and $36.6 \%$. The maximum cluster sizes of these analyses were seven for RFLP and eight for VNTR (Table 1, 4). The HGDI values were similar (0.9971 for RFLP and 0.9966 for VNTR).

\section{DISCUSSION}

The search for more appropriate genetic markers for the discrimination of $M$. tuberculosis isolates has led to the evaluation and selection of additional VNTR loci designated ETRs, QUBs, Mtub and other loci (Kremer et al., 2005a; Le Flèche et al., 2002; Roring et al., 2002, 2004; Skuce et al., 2002; Smittipat et al., 2005; Warren et al., 2004). For discriminating Beijing strains, a high efficiency of VNTR loci showing hyper-variability, such as some QUBs, has been reported (Kam et al., 2006; Kremer et al., 2005b; Nikolayevskyy et al., 2006; Surikova et al., 2005). The proposed optimized MIRU-VNTR typing excludes these loci because of their size allele designation and hyper-variability (Supply et al., 2006). In our data, VNTR 2163a and VNTR 2163b could not be amplified from 13 and 7 isolates (5.3 and $2.9 \%$ of strains), respectively. Moreover, 59 isolates $(24.3 \%)$ had copy numbers greater than 16 for VNTR 3232 (Table 3). These results pose a problem for the reliable sizing of amplicons and efficient allele assignment. However, in agreement to the results obtained, VNTR analyses of hyper-variable loci might be useful in some cases to discriminate W-Beijing strains that possess high clonality. Oelemann et al. (2006) used a combination of novel optimized VNTR and spoligotyping, which might be inappropriate for discrimination of strains of Beijing family.

Our data showed that 193 isolates ( $79.4 \%$ of the strains) were Beijing family strains, suggesting that such strains are prevalent in Japan, as in other East Asian areas (Bifani et al., 2002; Chan et al., 2001; van Soolingen et al., 1995). The discrimination by 12-loci MIRU-VNTR was low in Osaka because of the high prevalence of Beijing strains. This result indicates that, at least, 12-locus MIRU-VNTR is of limited value as a genotyping method of TB in Japan. We selected four QUB loci (VNTR 3232, VNTR 2163a, VNTR 2163b and VNTR 1982) that offered high allelic diversity, within the cluster, to adapt MIRU-VNTR analysis as a promising genotyping method for TB in Japan. The allelic diversity of these loci was consistent with that described in other reports related to analyses of Beijing strains (Kam et al., 2006; Kremer et al., 2005b; Nikolayevskyy et al., 2006; Surikova et al., 2005).

Table 4. The discriminatory power of 16-loci VNTR [MIRU + QUB(4)] in 243 M. tuberculosis strains from Osaka City

\begin{tabular}{|lccccc|}
\hline Typing method & $\begin{array}{c}\text { Total no. of type } \\
\text { patterns }\end{array}$ & $\begin{array}{c}\text { No. of unique } \\
\text { types }\end{array}$ & $\begin{array}{c}\text { No. of } \\
\text { clusters }\end{array}$ & $\begin{array}{c}\text { No. of clustered } \\
\text { isolates (\%) }\end{array}$ & $\begin{array}{c}\text { Maximum no. of } \\
\text { isolates in a cluster }\end{array}$ \\
\hline MIRU + QUB(4) VNTR $\dagger$ & 188 & 154 & 34 & $89(36.6)$ & 8 \\
\hline
\end{tabular}

${ }^{\star}$ HGDI was calculated as described by Hunter \& Gaston (1988).

†QUB(4) VNTR included QUB-11a, QUB-11b, QUB-18 and QUB-3232. According to new nomenclature, these are equivalent to VNTR 2163a, VNTR 2163b, VNTR 1982 and VNTR 3232, respectively. 
The QUB(4) loci were highly discriminatory in the 57 MIRU-identical clustered Beijing isolates, the performance was almost equivalent to that of IS6110 RFLP. The clusters A, B and C in Fig. 1 showed only one band difference in IS6110 RFLP. These clusters are therefore likely to represent transmission clusters. These clusters were also consistent with MIRU(12)+QUB(4) defined clusters, suggesting the usefulness of the addition of QUB $(4)$ VNTR typing in defining transmission clusters. In all of the 243 strains, the cluster analysis and the discriminatory power of MIRU(12) +QUB(4) were comparable to that of IS6110 RFLP (Table 4). These results demonstrated that the VNTR method can be used as an alternative for IS6110 RFLP typing in Japan.

Our approach for evaluating an alternative VNTR set, as a genotyping marker system for $\mathrm{TB}$ surveillance, was the addition of optional loci to a standard loci set to supplement its low discriminatory power for Beijing family strains. Such a strategy might facilitate both international comparison of epidemiological information and local discrimination of endemic isolates such as strains of the Beijing family.

\section{ACKNOWLEDGEMENTS}

The authors are grateful to A. Tamaru, Department of Microbiology, Osaka Prefectural Institute of Public Health, Osaka, Japan, for assistance with DNA electrophoresis using $i$-chip SV1210 apparatus. This work was supported by grants from the Japanese Ministry of Health, Labour and Welfare (Research on Emerging and Re-emerging Infectious Diseases, Health Sciences Research Grants), Ministry of Education, Culture, Sports, Science and Technology, Ministry of the Environment (Global Environment Research Fund), United States Japan Cooperative Medical Science Program against Tuberculosis and Leprosy, and Daido Life Welfare Foundation and Ohyama Health Foundation.

\section{REFERENCES}

Bifani, P. J., Mathema, B., Kurepina, N. E. \& Kreiswirth, B. N. (2002). Global dissemination of the Mycobacterium tuberculosis W-Beijing family strains. Trends Microbiol 10, 45-52.

Blackwood, K. S., Wolfe, J. N. \& Kabani, A. M. (2004). Application of mycobacterial interspersed repetitive unit typing to Manitoba tuberculosis cases: can restriction fragment length polymorphism be forgotten? J Clin Microbiol 42, 5001-5006.

Cave, M. D., Eisenach, K. D., McDermott, P. F., Bates, J. H. \& Crawford, J. T. (1991). IS6110: conservation of sequence in the Mycobacterium tuberculosis complex and its utilization in DNA fingerprinting. Mol Cell Probes 5, 73-80.

CDC (2004). Handbook for TB Controllers, Epidemiologists, Laboratorians, and Other Program Staff. National TB Controllers Association/CDC Advisory Group on Tuberculosis Genotyping. Atlanta, GA: CDC. http://www.cdc.gov/nchstp/tb/genotyping/toc. htm

Chan, M. Y., Borgdorff, M., Yip, C. W., de Haas, P. E., Wong, W. S., Kam, K. M. \& Van Soolingen, D. (2001). Seventy percent of the Mycobacterium tuberculosis isolates in Hong Kong represent the Beijing genotype. Epidemiol Infect 127, 169-171.
Cowan, L. S., Diem, L., Monson, T., Wand, P., Temporado, D., Oemig, T. V. \& Crawford, J. T. (2005). Evaluation of a two-step approach for large-scale, prospective genotyping of Mycobacterium tuberculosis isolates in the United States. J Clin Microbiol 43, 688-695.

Frothingham, R. \& Meeker-O'Connell, W. A. (1998). Genetic diversity in the Mycobacterium tuberculosis complex based on variable numbers of tandem DNA repeats. Microbiology 144, 1189-1196.

Glynn, J. R., Whiteley, J., Bifani, P. J., Kremer, K. \& van Soolingen, D. (2002). Worldwide occurrence of Beijing/W strains of Mycobacterium tuberculosis: a systematic review. Emerg Infect Dis 8, 843-849.

Hunter, P. R. \& Gaston, M. A. (1988). Numerical index of the discriminatory ability of typing systems: an application of Simpson's index of diversity. J Clin Microbiol 26, 2465-2466.

Kam, K. M., Yip, C. W., Tse, L. W., Leung, K. L., Wong, K. L., Ko, W. M. \& Wong, W. S. (2006). Optimization of variable number tandem repeat typing set for differentiating Mycobacterium tuberculosis strains in the Beijing family. FEMS Microbiol Lett 256, 258-265.

Kamerbeek, J., Schouls, L., Kolk, A., van Agterveld, M., van Soolingen, D., Kuijper, S., Bunschoten, A., Molhuizen, H., Shaw, R. \& other authors (1997). Simultaneous detection and strain differentiation of Mycobacterium tuberculosis for diagnosis and epidemiology. J Clin Microbiol 35, 907-914.

Kremer, K., van Soolingen, D., Frothingham, R., Haas, W. H., Hermans, P. W., Martin, C., Palittapongarnpim, P., Plikaytis, B. B., Riley, L. W. \& other authors (1999). Comparison of methods based on different molecular epidemiological markers for typing of $\mathrm{Myco}$ bacterium tuberculosis complex strains: interlaboratory study of discriminatory power and reproducibility. J Clin Microbiol 37, 2607-2618.

Kremer, K., Glynn, J. R., Lillebaek, T., Niemann, S., Kurepina, N. E., Kreiswirth, B. N., Bifani, P. J. \& van Soolingen, D. (2004). Definition of the Beijing/W lineage of Mycobacterium tuberculosis on the basis of genetic markers. J Clin Microbiol 42, 4040-4049.

Kremer, K., Arnold, C., Cataldi, A., Gutierrez, M. C., Haas, W. H., Panaiotov, S., Skuce, R. A., Supply, P., van der Zanden, A. G. \& van Soolingen, D. (2005a). Discriminatory power and reproducibility of novel DNA typing methods for Mycobacterium tuberculosis complex strains. J Clin Microbiol 43, 5628-5638.

Kremer, K., Au, B. K., Yip, P. C., Skuce, R., Supply, P., Kam, K. M. \& van Soolingen, D. (2005b). Use of variable-number tandem-repeat typing to differentiate Mycobacterium tuberculosis Beijing family isolates from Hong Kong and comparison with IS6110 restriction fragment length polymorphism typing and spoligotyping. J Clin Microbiol 43, 314-320.

Le Flèche, P., Fabre, M., Denoeud, F., Koeck, J. L. \& Vergnaud, G. (2002). High resolution, on-line identification of strains from the Mycobacterium tuberculosis complex based on tandem repeat typing. BMC Microbiol 2, 37.

Mazars, E., Lesjean, S., Banuls, A. L., Gilbert, M., Vincent, V., Gicquel, B., Tibayrenc, M., Locht, C. \& Supply, P. (2001). High-resolution minisatellite-based typing as a portable approach to global analysis of Mycobacterium tuberculosis molecular epidemiology. Proc Natl Acad Sci U S A 98, 1901-1906.

Molhuizen, H. O., Bunschoten, A. E., Schouls, L. M. \& van Embden, J. D. (1998). Rapid detection and simultaneous strain differentiation of Mycobacterium tuberculosis complex bacteria by spoligotyping. Methods Mol Biol 101, 381-394.

Nikolayevskyy, V., Gopaul, K., Balabanova, Y., Brown, T., Fedorin, I. \& Drobniewski, F. (2006). Differentiation of tuberculosis strains in a population with mainly Beijing-family strains. Emerg Infect Dis 12, $1406-1413$.

Oelemann, M. C., Diel, R., Vatin, V., Haas, W., Rusch-Gerdes, S., Locht, C., Niemann, S. \& Supply, P. (2006). Assessment of an 
optimized mycobacterial interspersed repetitive-unit-variable-number tandem-repeat typing system combined with spoligotyping for population-based molecular epidemiology studies of tuberculosis. $J$ Clin Microbiol 45, 691-697.

Roring, S., Scott, A., Brittain, D., Walker, I., Hewinson, G., Neill, S. \& Skuce, R. (2002). Development of variable-number tandem repeat typing of Mycobacterium bovis: comparison of results with those obtained by using existing exact tandem repeats and spoligotyping. $J$ Clin Microbiol 40, 2126-2133.

Roring, S., Scott, A. N., Glyn Hewinson, R., Neill, S. D. \& Skuce, R. A. (2004). Evaluation of variable number tandem repeat (VNTR) loci in molecular typing of Mycobacterium bovis isolates from Ireland. Vet Microbiol 101, 65-73.

Skuce, R. A., McCorry, T. P., McCarroll, J. F., Roring, S. M., Scott, A. N., Brittain, D., Hughes, S. L., Hewinson, R. G. \& Neill, S. D. (2002). Discrimination of Mycobacterium tuberculosis complex bacteria using novel VNTR-PCR targets. Microbiology 148, 519-528.

Smittipat, N., Billamas, P., Palittapongarnpim, M., Thong-On, A., Temu, M. M., Thanakijcharoen, P., Karnkawinpong, O. \& Palittapongarnpim, P. (2005). Polymorphism of variable-number tandem repeats at multiple loci in Mycobacterium tuberculosis. J Clin Microbiol 43, 5034-5043.

Sun, Y. J., Bellamy, R., Lee, A. S., Ng, S. T., Ravindran, S., Wong, S. Y., Locht, C., Supply, P. \& Paton, N. I. (2004). Use of mycobacterial interspersed repetitive unit-variable-number tandem repeat typing to examine genetic diversity of Mycobacterium tuberculosis in Singapore. J Clin Microbiol 42, 1986-1993.

Supply, P., Mazars, E., Lesjean, S., Vincent, V., Gicquel, B. \& Locht, C. (2000). Variable human minisatellite-like regions in the Mycobacterium tuberculosis genome. Mol Microbiol 36, 762-771.
Supply, P., Lesjean, S., Savine, E., Kremer, K., van Soolingen, D. \& Locht, C. (2001). Automated high-throughput genotyping for study of global epidemiology of Mycobacterium tuberculosis based on mycobacterial interspersed repetitive units. J Clin Microbiol 39, 3563-3571.

Supply, P., Allix, C., Lesjean, S., Cardoso-Oelemann, M., RuschGerdes, S., Willery, E., Savine, E., de Haas, P., van Deutekom, H. \& other authors (2006). Proposal for standardization of optimized mycobacterial interspersed repetitive unit-variable-number tandem repeat typing of Mycobacterium tuberculosis. J Clin Microbiol 44, 4498-4510.

Surikova, O. V., Voitech, D. S., Kuzmicheva, G., Tatkov, S. I., Mokrousov, I. V., Narvskaya, O. V., Rot, M. A., van Soolingen, D. \& Filipenko, M. L. (2005). Efficient differentiation of Mycobacterium tuberculosis strains of the W-Beijing family from Russia using highly polymorphic VNTR loci. Eur J Epidemiol 20, 963-974.

van Embden, J. D., Cave, M. D., Crawford, J. T., Dale, J. W., Eisenach, K. D., Gicquel, B., Hermans, P., Martin, C., McAdam, R. \& other authors (1993). Strain identification of Mycobacterium tuberculosis by DNA fingerprinting: recommendations for a standardized methodology. J Clin Microbiol 31, 406-409.

van Soolingen, D., Qian, L., de Haas, P. E., Douglas, J. T., Traore, H., Portaels, F., Qing, H. Z., Enkhsaikan, D., Nymadawa, P. \& van Embden, J. D. (1995). Predominance of a single genotype of Mycobacterium tuberculosis in countries of east Asia. J Clin Microbiol 33, 3234-3238.

Warren, R. M., Victor, T. C., Streicher, E. M., Richardson, M., van der Spuy, G. D., Johnson, R., Chihota, V. N., Locht, C., Supply, P. \& van Helden, P. D. (2004). Clonal expansion of a globally disseminated lineage of Mycobacterium tuberculosis with low IS6110 copy numbers. J Clin Microbiol 42, 5774-5782. 